close agreement with Mr. Petch's determination. The X-ray photograph of a tungsten powder containing a proportion of the $\alpha$-phase is also reproduced in the illustration.

It is apparent from the results described by $\mathrm{Mr}$. Petch and ourselves that the $\alpha$-form of tungsten may be produced in several ways besides that of electrolytic extraction, namely, by sputtering, by volatilization or evaporation, and, in certain circumstances, by reduction of oxide in hydrogen. The temperature of deposition (or reduction) is probably an important factor in determining the proportion of $\alpha$-tungsten formed, but the evidence from the examination of volatilized films and reduced powders suggests that impurities such as phosphorus and thoria may also take some part.

Research Laboratories of

H. P. Rooksby.

The General Electric Company, Ltd., Wembley. July 26.

\section{Thermal Fatigue of Metals}

Is our recent communication ${ }^{1}$ the photographs as reproduced were placed in the wrong order, so the

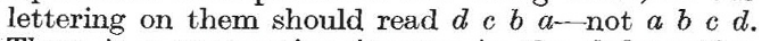
There is a progressive increase in the deformation and in the grain boundary migration as the number of thermal cycles is increased.

The deformation, as indicated by the formation of slip lines, and the grain boundary migration, are shown more clearly in the accompanying photograph. This illustrates at two different magnifications the numerous slip lines and the grain boundary migration produced in a cadmium specimen after ten very slow cycles between $30^{\circ} \mathrm{C}$. and $150^{\circ} \mathrm{C}$. The electrolytically polished specimen, which initially was almost free from slip lines, was immersed in an oil bath, and was heated and cooled with it, the duration of a cycle being approximately seven hours. This was done in order to avoid internal stresses due to rapid temperature changes.

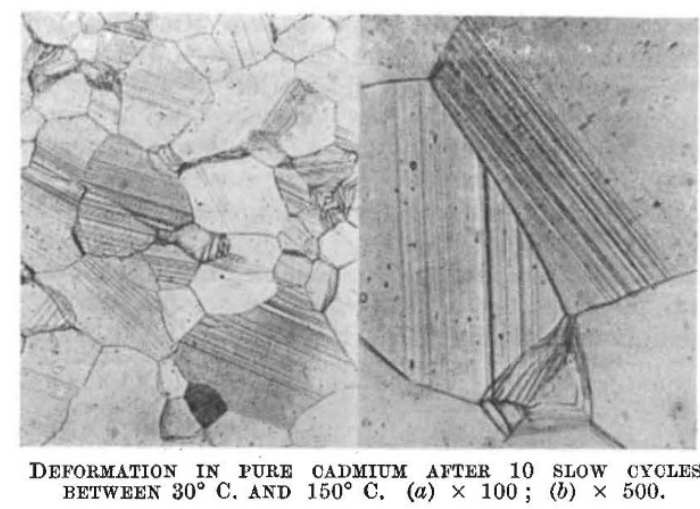

Experiments carried out since our earlier communication have confirmed the view that the deformation observed after cyclic thermal treatment is due to the anisotropy of thermal expansion in the crystals of non-cubic metals.

X-ray back-reflexion photographs show that the lattice distortions produced by the deformation re. main in the metal and accumulate as the number of cycles is increased. This is the case for zinc, cadmium and even tin, although in the latter metal the outward signs of deformation are less than in zine and cadmium. In some cases, recovery occurs after a large number of cycles.

W. BOAS.

R. W. K. HONEYCOMBE.

Council for Scientific and Industrial Research, Lubricants and Bearings Section, University of Melbourne. June 22.

${ }^{1}$ Nature, 153, 494 (1944).

\section{Relationship between Coercive Force and Carbon Content of Plain Carbon Steels}

THE straight-line relation between the coercive force and the carbon content of plain carbon steels has been observed by many investigators. The question arises how far can the relation be carried. The data published by authorities such as Benedicks, Metallografiska Institutet, Stockholm, and Cheney, U.S. Bureau of Standards, have been collected in the International Critical Tables'. They used samples containing very small amounts of silicon, manganese, sulphur and other impurities. If their results are plotted we obtain a straight line as shown in Fig. 1.

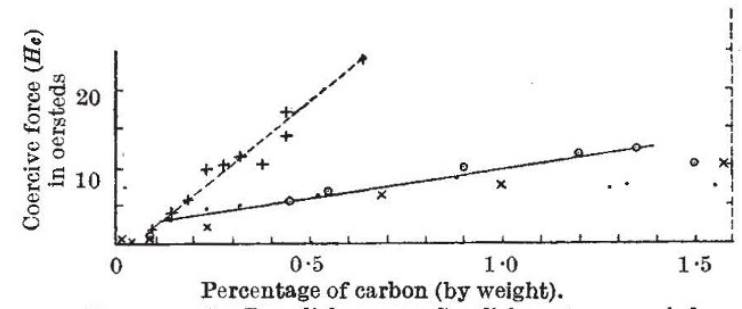

., Chency ; $\odot$, Benedicks $; 5^{\circ} \stackrel{\times}{\mathrm{C}}$, Gumlich; + , annealed, Fig. 1.

Carbon enters into iron forming pearlite, a structure which can be distinguished from ferrite (iron) under the microscope, when the concentration is more than 0.008 per cent. The amount of pearlite increases as the carbon in iron is increased. If we plot the percentages of pearlite against the coercive forces of the annealed specimens, we obtain the result shown in Fig. 2. The formation of pearlite is complete when the carbon content reaches 0.83 per cent; that is, the eutectoid. After passing the eutectoid, cementite comes into play.

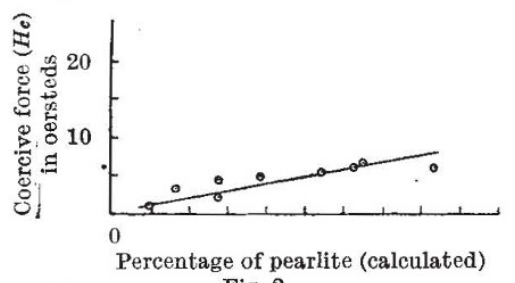

Fig. 2.

When the steel is in a quenched state, the relationship of the coercive force becomes more complicated. It depends on the quenching temperature and the cooling medium. The results from Gumlich are redrawn in Fig. 3.

Recently various applications have been found in this relationship between the coercive force and the carbon content. The instrument, which is known as 